\title{
Biologia reprodutiva do camarão-branco, Penaeus schmitti (Decapoda, Penaeidae), na Área de Proteção Ambiental do Delta do Parnaíba-MA
}

\author{
Reproductive biology of the white shrimp, Penaeus schmitti (Decapoda, Penaeidae) in the \\ Environmental Protection Area of Delta do Parnaíba-MA
}

Biología reproductiva del camarón blanco Penaeus schmitti (Decapoda, Penaeidae) en el Área de Protección Ambiental del Delta del Parnaíba-MA

\section{Resumo}

Penaeus schmitti é uma espécie de grande importância para o estado do Maranhão, porém estudos acerca de sua biologia são pouco conhecidos no estado, o que torna essencial a realização de tais estudos, uma vez que estes auxiliam no manejo correto da espécie e consequentemente a preservação da mesma. Desta forma, este trabalho teve por objetivo caracterizar os aspectos reprodutivos de $P$. schmitti no município de Tutóia, Maranhão, através de análises macroscópicas das gônadas, da determinação do comprimento, da proporção sexual e primeira maturidade. Durante o período de um ano foram analisados 240 exemplares. As diferenças na proporção sexual foram testadas pelo teste do qui-quadrado $\left(\chi^{2}\right)$ ao nível de significância de 0,05 . Quanto a proporção sexual, constatou-se que as fêmeas tiveram maior representatividade que os machos, demonstrando assim, respetivamente, $57 \%$ e $43 \%$ do total amostrado. Ao realizar a análise da distribuição por classe de comprimento de $P$. schmitti, percebeu-se que as fêmeas se apresentaram maiores que os machos. Onde o comprimento médio das fêmeas foi de 11,08 cm e do machos 10,07 $\mathrm{cm}$. Não foi possível constatar o período reprodutivo da espécie devido à ausência de indivíduos em estádio maturo. O tamanho encontrado da primeira maturação de $P$. Schmitti foi de $9,49 \mathrm{~cm}$. Valor inferior aos que foram encontrados em outras regiões no nordeste do Brasil. Diante das informações obtidas são sugeridas medidas de gerenciamento e conservação da espécie visando a exploração racional do recurso.

Palavras-chave: Primeira maturação; Proporção sexual; Tutóia.

\section{Abstract}

Penaeus schmitti is a species of great importance to the state of Maranhão, but studies on its biology are little known in the state, which makes it essential to conduct such studies, since they assist in the correct management of the species and consequently its preservation. Thus, this work aimed to characterize the reproductive aspects of P. schmitti in Tutóia, Maranhão, through macroscopic analysis of the gonads, length determination, sex ratio and first maturity. During the period of one year, 240 specimens were analyzed. The differences in sex ratio were tested by the chi-square 
test $(\chi 2)$ at a significance level of 0.05 . Regarding sex ratio, it was found that females were more representative than males, thus demonstrating, respectively, $57 \%$ and $43 \%$ of the total sample. When analyzing the distribution by length class of P. schmitti, it was noticed that females were bigger than males. The average length of females was $11.08 \mathrm{~cm}$ and males, $10.07 \mathrm{~cm}$. It was not possible to determine the reproductive period of the species due to the absence of individuals in the mature stage. The size of the first maturity of P. schmitti was $9.49 \mathrm{~cm}$. This value was lower than those found in other regions in the northeast of Brazil. In view of the information obtained, management and conservation measures for the species are suggested, aiming at the rational exploitation of the resource.

Keywords: First maturation; Sex ratio; Tutóia.

\section{Resumen}

Penaeus schmitti es una especie importante para el estado de Maranhão, pero los estudios sobre su biología son poco conocidos en el estado, lo que hace imprescindible la realización de dichos estudios, ya que ayudan a la correcta gestión de la especie y consecuentemente a su preservación. Así, este trabajo tuvo como objetivo caracterizar los aspectos reproductivos de P. schmitti en el municipio de Tutóia, Maranhão, a través del análisis macroscópico de las gónadas, determinación de la longitud, la proporción sexual y la primera madurez. Durante el período de un año, se analizaron 240 especímenes. Las diferencias en la proporción de sexos se comprobaron mediante la prueba de chicuadrado $(\chi 2)$ En cuanto a la proporción de sexos, se comprobó que las mujeres eran más representativas que los hombres, con un $57 \%$ y un $43 \%$, respectivamente, del total de la muestra. Al analizar la distribución por clase de longitud de P. schmitti, se observó que las hembras eran más grandes que los machos. La longitud media de las hembras fue de $11,08 \mathrm{~cm}$ y la de los machos de $10,07 \mathrm{~cm}$. No fue posible determinar el período reproductivo de la especie debido a la ausencia de individuos en la etapa madura. La talla de primera madurez de P. schmitti fue de 9,49 $\mathrm{cm}$. Este valor era inferior a los encontrados en otras regiones del noreste de Brasil. A la vista de la información obtenida, se sugieren medidas de gestión y conservación de la especie, encaminadas a la explotación racional del recurso.

Palabras clave: Primera maduración; Relación de sexos; Tutóia.

\section{Introdução}

Nos países em desenvolvimento, a atividade pesqueira tem importância incontestável como fonte de alimento e renda para milhares de famílias em situação de vulnerabilidade (FAO 2017, apud Mendes, 2020). Entre as espécies de maior importância econômica, devido ao seu elevado valor comercial e boa aceitação no mercado, estão os camarões marinhos. As famílias de camarões com maior interesse de pesca no litoral brasileiro são os peneídeos (Freire, 2019). Inúmeras pessoas dependem, direta ou indiretamente, da pesca desse camarão no nordeste brasileiro: são homens, mulheres e crianças que pescam, beneficiam e comercializam esses camarões (Santos et al 2006).

Na família Penaeidae está inserido Penaeus schmitti conhecido popularmente como camarão-branco, ele apresenta importância ecológica e econômica significativa (Cordeiro, 2020) e está entre as principais espécies comercialmente importantes no Brasil (Lopes, 2012). Os camarões peneídeos apresentam grande importância comercial, por esse motivo, ao longo das regiões costeiras são intensamente pescados, sendo capturados tanto em estrato juvenil como no seu estrato adulto. Nesse contexto, os impactos são preocupantes, pois, o elevado esforço de pesca nos dois estratos prejudica o fechamento de seu ciclo de vida e é seu principal fator de decréscimo em abundância (Boss et al., 2016).

O camarão-branco é uma espécie muito valorizada no município de Tutóia, município este que faz parte da área de proteção ambiental do Delta do Parnaíba, que abrange três estados do Nordeste: Piauí, Maranhão e Ceará. Um dos objetivos desta área é a proteção de sua fauna. P. schmitti é ecologicamente e economicamente importante para o estado do Maranhão, porém os estudos acerca de sua biologia reprodutiva são desconhecidos ou desatualizados no estado, o que torna essencial esta pesquisa, pois estudos realizados sobre a dinâmica reprodutiva de camarões, além de contribuírem para um melhor conhecimento sobre o seu ciclo de vida, ajudam na adequabilidade de plano de manejo das espécies explotadas pela pesca. Os estudos sobre a reprodução são relevantes para a formulação de políticas públicas, mas especificamente através da definição de um período de defeso, na regularização dos tamanhos de malhas apropriadas para não se capturar indivíduos abaixo do tamanho de primeira maturação sexual e delimitação de zonas protegidas para reprodução e berçário. Conhecer a biologia 
reprodutiva de camarões é uma ferramenta importante para avaliar os potenciais candidatos à aquicultura bem como estratégias para a preservação da biodiversidade (Mossolin \& Bueno, 2002).

Desta maneira, objetivou-se caracterizar a atividade reprodutiva de $P$. schmitti, espécie de importância comercial pertencente a Área de Proteção Ambiental do Delta do Parnaíba, contribuindo assim para um melhor conhecimento da biologia e sustentabilidade desse camarão.

\section{Metodologia}

A pesquisa foi realizada na Área de Proteção Ambiental do Delta do Parnaíba. A APA protege áreas importantes como Tutóia, Melancieiras, Caju e Canárias, pequenos igarapés e o mangue, para a reprodução de espécies, funcionando como um berçário que garante a reposição dos estoques pesqueiros (ICMBIO, 2020). A área escolhida para a realização do trabalho foi o município de Tutóia (2o 45'44”S; 42o 16’28”W) que se localiza no Litoral Oriental do Maranhão, especificamente entre os Lençóis Maranhenses e o Delta do Parnaíba, na divisa com o estado do Piauí, em frente a Baía de Tutóia (IBGE, 2010).

O litoral de Tutóia apresenta feições eólicas relativamente próximas a linha de costa, resultantes da ação conjugada de ondas, correntes costeiras, ação fluvial, variações do nível do mar e fatores climáticos que contribuíram para a configuração da atual paisagem costeira (Silva, 2018.). O clima da região é tropical (AW’) subúmido com dois períodos bem definidos: um chuvoso que vai de janeiro a junho com medias mensais superiores a $237 \mathrm{~mm}$ e outro seco, correspondente aos meses de julho a dezembro (Jornal Do Tempo, 2011).

Figura 1. Mapa de localização do Município de Tutóia.

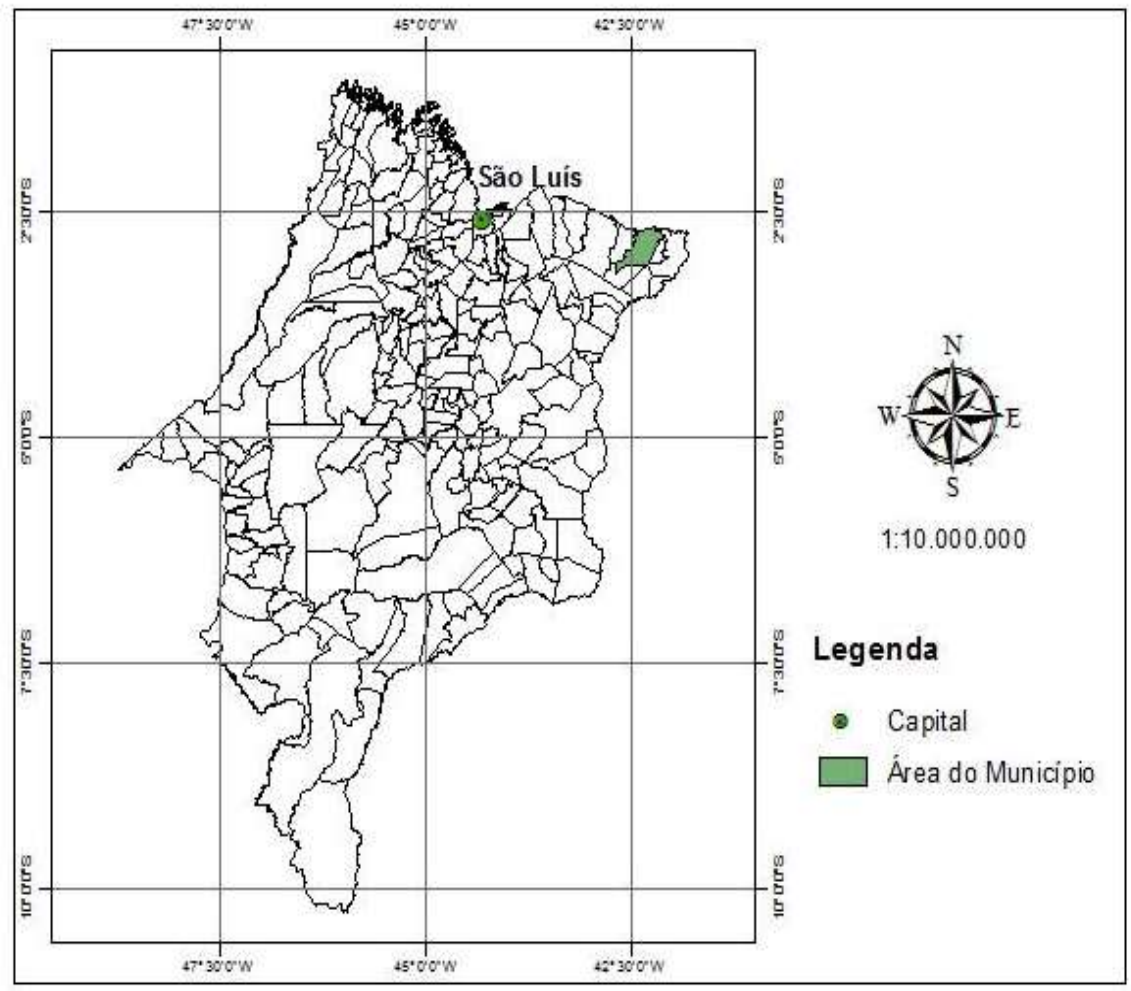

Fonte: CPRM (2011). 


\section{Resultados}

Foram analisados de forma padronizada 20 exemplares ao mês, totalizando 240 exemplares. As fêmeas foram responsáveis pela maior representatividade (Figura 2). A proporção sexual foi de 1,33:1 (fêmeas e machos), representando assim, respectivamente, $57 \%$ e $43 \%$ do total amostrado. O teste de qui-quadrado $\left(\chi^{2}=15,4 ; p<0,05\right)$ mostrou que houve diferença significativa entre os sexos.

Figura 2. Proporção sexual mensal de Penaeus schimitti, capturado no município de Tutoia, MA, no período de janeiro a dezembro de 2018.

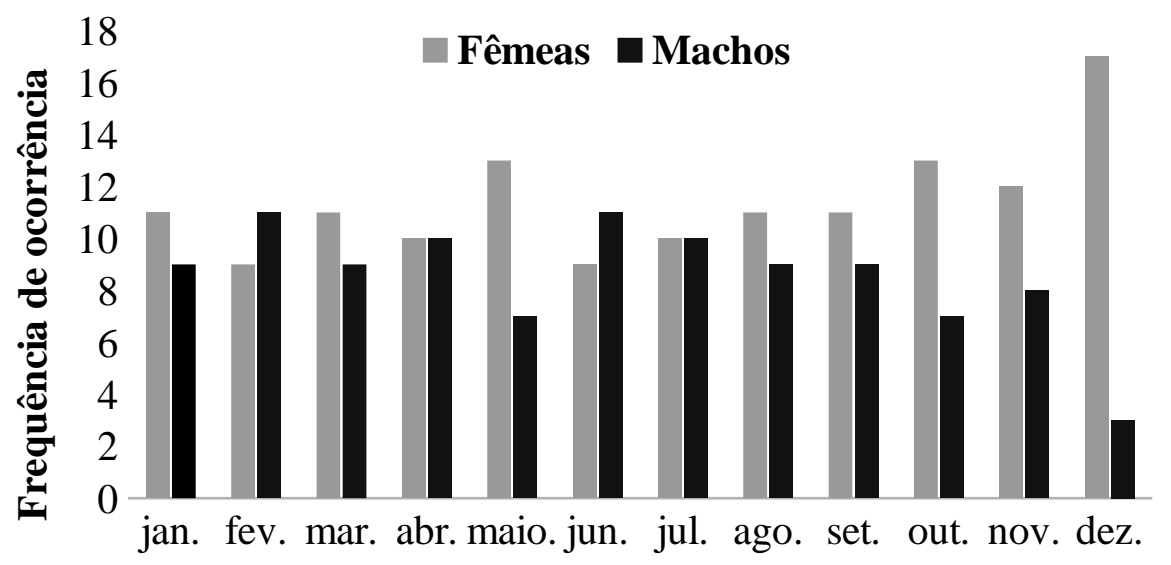

\section{Meses}

Fonte: Autores.

A variação do comprimento corporal total para as fêmeas $(7$ a $16 \mathrm{~cm})$ foi maior em comparação com os machos $(6,2$ a $15,6 \mathrm{~cm}$ ) (Figura 3).

Figura 3. Distribuição da frequência relativa por classe de comprimento corporal total de fêmeas e machos de Penaeus schimitti, capturados no município de Tutóia, MA, no período de janeiro a dezembro de 2018.

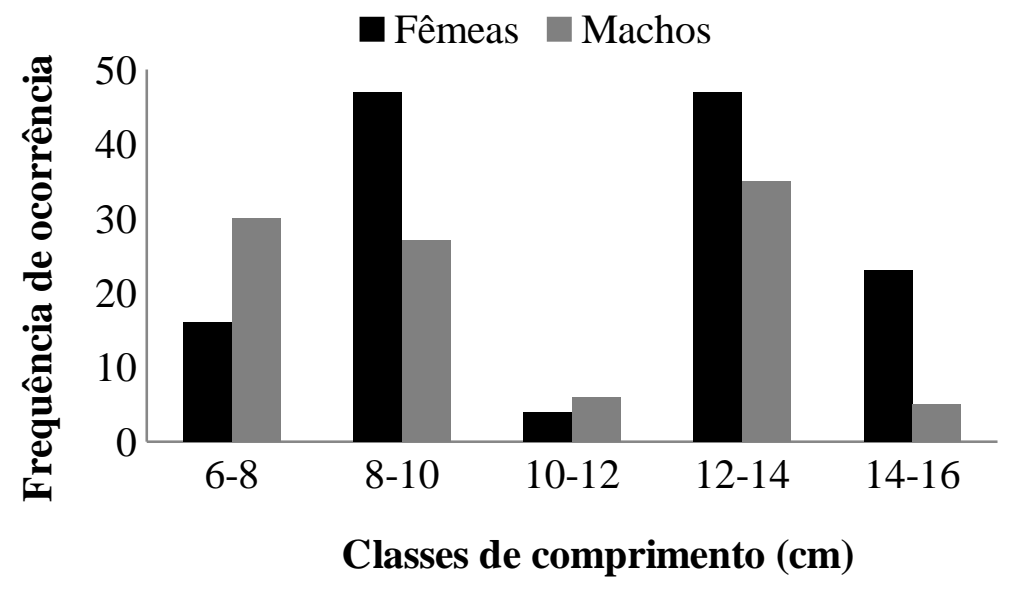

Fonte: Autores.

Das cinco classes de comprimento descritas no presente trabalho, as fêmeas foram dominantes em três delas. A partir do teste do $\chi^{2}$, indicou uma significativa diferença entre os sexos $(\chi 2=23,29 ; \mathrm{p}<0,05)$ (Tabela 1). 
Tabela 1 Valores do $\left(\chi^{2}\right)$ qui-quadrado por classe de comprimento total $(\mathrm{cm})$ para Penaeus schimitt capturados no município de Tutóia, MA, no período de janeiro a dezembro de 2018.

\begin{tabular}{cccccc}
\hline Classes & Fêmeas & Machos & Total & Fe & $\chi^{\mathbf{2}}$ \\
\hline $06-08$ & 16 & 30 & 46 & 23 & 4.26 \\
$08-10$ & 47 & 27 & 74 & 37 & 5.40 \\
$10-12$ & 4 & 6 & 10 & 5 & 0,4 \\
$12-14$ & 47 & 35 & 82 & 41 & 1,76 \\
$14-16$ & 23 & 5 & 28 & 14 & 11,57 \\
\hline Total geral & $\mathbf{1 3 7}$ & $\mathbf{1 0 3}$ & $\mathbf{2 4 0}$ & $\mathbf{1 2 0}$ & $\mathbf{2 3 . 3 9}$ \\
\hline
\end{tabular}

Fonte: Autores.

\subsection{Caracterização dos estádios de maturação gonadal}

A maturação gonadal nessa espécie, assim como em outros grupos de crustáceos decápodes, é caracterizada por uma série de modificações anatômicas, sendo possível observar o aumento do volume das gônadas e mudança em sua coloração. De acordo com a análise da coloração e espessura das gônadas foram identificados três estágios de maturação gonadal: Imaturo, Em desenvolvimento e Vazio (Tabela 2).

Tabela 2. Classificação dos estádios de maturação gonadal de machos e fêmeas de Penaeus schmitti capturados no município de Tutóia, MA, no período de janeiro a dezembro de 2018.

\begin{tabular}{ccc}
\hline $\begin{array}{c}\text { Estádio de maturação } \\
\text { gonadal }\end{array}$ & Fêmea & Machos \\
\hline Imaturo & Os ovários apresentam-se finos e & Os testículos se apresentam bem \\
& transparentes & finos e transparentes \\
Em desenvolvimento & Os ovários assumem coloração & Os testículos se apresentam \\
& variada e de diferentes tonalidades de & esbranquiçados, porém ainda \\
& amarelo. & finos. \\
Vazio & A gônada apresenta-se flácida e de & - \\
& coloração esbranquiçada. & \\
\hline
\end{tabular}

Fonte: Autores.

Os exemplares estavam distribuídos nas seguintes proporções: em desenvolvimento $(58,34 \%)$, imaturo $(31,25 \%)$ e vazio $(10,41 \%)$. Ao observar os percentuais dos estádios maturacionais dos exemplares, nota-se a predominância de indivíduos em desenvolvimento. Ao analisar os sexos separadamente, percebe-se que para ambos os sexos houve predomínio dos indivíduos nesse estádio. A maior proporção de indivíduos vazios foi de fevereiro a abril e Julho a novembro. Não foi possível constatar o período reprodutivo da espécie devido à ausência de indivíduos em estádio maturo (Figura 4). 
Figura 4. Estádios de maturação gonadal de machos e fêmeas de Penaeus schmitti capturados no município de Tutóia, MA, no período de janeiro a dezembro de 2018. *IM (imaturo), ED (em desenvolvimento), VZ (vazio).

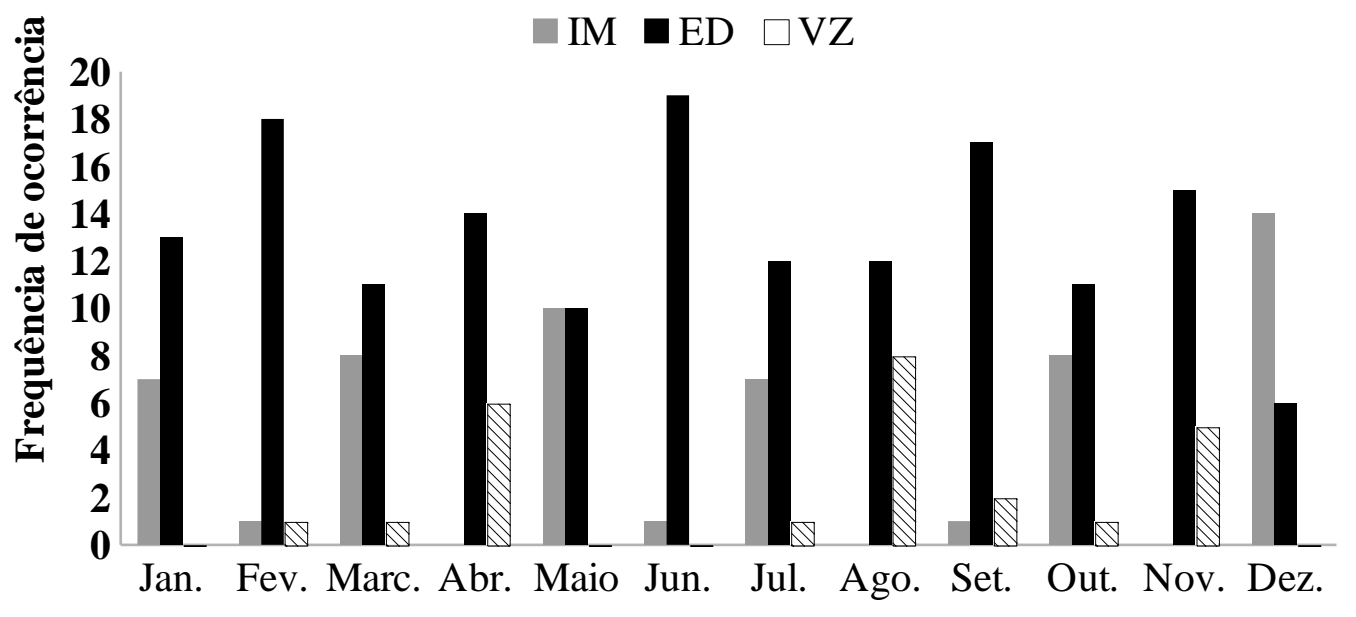

\section{Meses}

Fonte: Autores.

\subsection{Primeira maturação}

O tamanho estimado da primeira maturação gonadal o para $P$. schmitti foi de $9,49 \mathrm{~cm}$ (Figura 5). Este é o tamanho mínimo de captura para $P$. schmitti, onde $50 \%$ da população está apta a se reproduzir. Isso representa um aspecto importante na conservação e manejo adequado da referida espécie.

Figura 5. Tamanho da primeira maturação gonadal de Penaeus schmitti capturados no município de Tutóia, MA, no período de janeiro a dezembro de 2018.

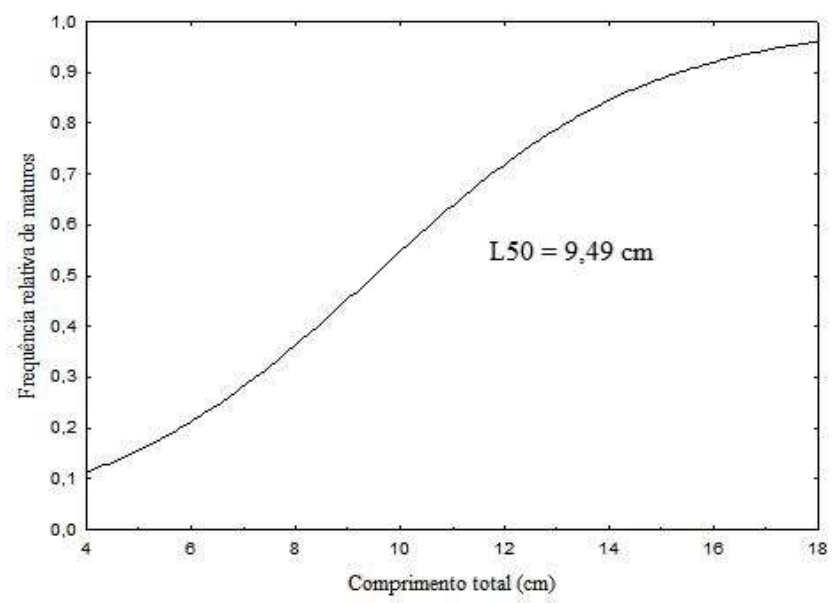

Fonte: Autores.

\section{Discussão}

A proporção sexual de 1,33:1 (fêmeas e machos) observada no presente estudo está dentro da faixa relatada para populações de camarão-branco em diferentes localidades do Nordeste do Brasil. Peixoto et al (2018) encontraram a proporção de 1,38:1 (fêmeas e machos) na costa de Pernambuco. Já Craveiro (2019) registrou a predominância de fêmeas com a 
proporção de 1,30:1 na Paraíba. Para estes autores, este índice elevado de fêmeas pode ser um forte indício de uma área de desova ou acasalamento para populações de $P$. schimitti.

A predominância das fêmeas também foi relatada na região Sudeste. Santos et. al., (2008) encontraram nas regiões estuarina e marinha da baixada santista, uma maior proporção de indivíduos do sexo feminino. Santos (2018) ao realizar a análise da estrutura populacional desta espécie nesta mesma localidade, encontrou a proporção sexual de $72 \%$ de fêmeas e $28 \%$ de machos (2,5:1), na área de mar aberto, e 75,11\% de fêmeas e 24,89\% de machos (3:1), na região do estuário.

Segundo Wenner, (1972) conforme citado Carvalho (2013), razões sexuais diferentes de 1:1 são comumente observadas entre os crustáceos, tal fato pode ser ocasionado por diferenças no seu ciclo de vida, migração, taxa de mortalidade e de crescimento diferencial entre machos e fêmeas e outras diferenças comportamentais.

Outra justificativa para a maior incidência de fêmeas é o fato de que os machos tendem a se enterrar mais do que as fêmeas no substrato, o que pode dificultar assim a sua captura (Santos \& Freitas, 2004), já as fêmeas, ficam mais exposta, por terem períodos mais longos de procura por alimento, a fim de obter energia para a maturação, se tornam mais vulneráveis a pesca (Craveiro, 2019).

O presente estudo também indicou que, apesar de ambos os sexos apresentarem tamanho médio relativamente próximos, as fêmeas atingiram tamanhos máximos maiores. O que também foi relatado anteriormente para populações de $P$. schimitti nos estudos de Santos (2010) em Coruripe; Bochini (2012) na região de Ubatuba; Freire (2019) e Peixoto (2018) em Pernambuco e Craveiro (2019) na Paraíba. Esta diferença de tamanho pode ocorrer devido ao fato das fêmeas precisarem de um espaço na cavidade cefalotoráxica onde estão situadas as gônadas, auxiliando assim no desenvolvimento das mesmas e consequentemente contribuindo na reprodução da espécie (Dall et al 1990. APUD Peixoto 2018).

As fêmeas dos camarões Penaeidae sempre alcançam comprimento total superior ao dos machos. (Boschi 1969). Indivíduos menores foram predominantes nos meses de setembro, outubro, junho e julho, concordando com Silva et al (2018) que ao realizarem um estudo sobre a dinâmica reprodutiva do camarão-branco no litoral sul de Pernambuco, registraram a ocorrência dos menores indivíduos nesse mesmo período. Segundo os autores, isso é indício de um possível recrutamento para esses meses. O que também está de acordo com a possível época de desova observada no presente estudo de fevereiro a abril e Julho a novembro

Quanto aos estádios maturacionais encontrados, os resultados corroboram com Santos et. al (2010) no município de Coruripe, Alagoas, onde a maioria das fêmeas encontradas apresentavam-se em desenvolvimento. De acordo com os autores, o elevado índice de fêmeas em desenvolvimento somado ao predomínio das mesmas em relação aos machos, pode ser um forte indício de uma área de acasalamento para a espécie.

No presente trabalho o período em que mais se encontrou indivíduos vazios foi de fevereiro a abril e julho a novembro. Esses resultados são similares a outros estudos realizados no Nordeste do Brasil. Calazans (2013) analisando a dinâmica reprodutiva do $P$. schmitti no litoral sul de Pernambuco, registrou a presença de indivíduos vazios no período de agosto e novembro. Peixoto (2018) registrou uma grande incidência dos indivíduos vazios no período de agosto a novembro e fevereiro a março, também em Pernambuco. Para os autores este é um forte indício de que esses meses estão relacionados a época de desova da espécie e determinaram duas épocas de desova: fevereiro a abril e agosto a novembro.

O comprimento encontrado para a primeira maturação foi de $9,49 \mathrm{~cm}$, tal resultado é inferior aos valores descritos em outros trabalhos sobre a biologia reprodutiva dessa espécie. Machado (2009) em seus estudos sobre o camarão branco na região sul do Brasil, estimou o tamanho de primeira maturação em 15,2 cm, já Peixoto et al. (2018) estimou a primeira maturação em 14,2 cm e Calazans (2013) estabeleceu o tamanho de 13,6 cm.

Essa diferença encontrada no comprimento de primeira maturidade pode ocorrer devido à maior temperatura das águas costeiras da região Nordeste, o que poderia aumentar a taxa de crescimento e promover uma maturação prematura de $P$. 
schmitti nesta região (Peixoto et al., 2018). O aumento da pressão da pesca também pode alterar as características da história de vida, como crescimento e tamanho da maturidade sexual. (Lappalainen et al. 2016 APUD Peixoto et al., 2018).

\section{Considerações Finais}

Não houve incidência de indivíduos maturos, tornando inviável constatar o período reprodutivo dessa espécie no município de Tutóia.

A proporção sexual de $P$. Schmitti foi de 1,33F:1M, a maior proporção de fêmeas, possivelmente indica que a área de estudo é uma área de acasalamento ou desova para populações de $P$. schimitti.

Os menores indivíduos registrados nos meses de setembro, outubro, junho e julho é um grande indício de um possível recrutamento para esses meses. O maior percentual de indivíduos vazios ocorreu nos meses de agosto e novembro, o que pode indicar ser esse o período de desova da espécie.

O tamanho da primeira maturação foi de $9,49 \mathrm{~cm}$. Analisando as classes de comprimento dos indivíduos capturados, percebe-se que a pesca no município de Tutóia captura, principalmente, indivíduos que já haviam alcançado a primeira maturação.

Assim, sugere-se que seja evitada a captura de indivíduos de P. Schmitti menores que o tamanho estimado e também se recomenda maior atenção nos meses de agosto, novembro e abril, pois há uma possibilidade da desova da espécie ser nesta época. Espera-se que os dados fornecidos auxiliem na compreensão do ciclo de vida de P. schimitti a fim de estabelecer políticas de manejo de recursos pesqueiros e preservação da espécie

\section{Agradecimentos}

Agradecemos ao Conselho Nacional de Desenvolvimento Cientifico e Tecnológico CNPq pela oportunidade da bolsa de iniciação científica e ao Laboratório de Pesca e Ecologia Aquática - LabPEA da Universidade Estadual do Maranhão e sua equipe de pesquisadores pelo grande apoio durante todas as etapas deste trabalho.

\section{Referências}

Bochini, G. L. (2012). Distribuição ecológica e estrutura populacional em escala espacial, temporal e anual do camarão-branco Litopenaeus schmitti (Burkenroad, 1936) (Dendrobranchiata: Penaeidae) na enseada de Ubatuba: 4 anos de estudo. (Dissertação de mestrado. Universidade Estadual Paulista)

Boschi, E. E. (1969). Estudio biológico pesquero del camarón Artemesia longinaris Bate de Mar del Plata. Boletín del instituto de Biología Marina, (18), 1-51.

Boos, H., Costa, R. C., Santos, R. A. F., Dias-Neto, J., Severino-Rodrigues, E., Rodrigues, L. F., \& Pinheiro, M. (2010). Avaliação dos camarões peneídeos (Decapoda: Penaeidae). Livro vermelho dos crustáceos do Brasil: Avaliação, 2014, 300-317.

Calazans, N. K. F. (2013) Dinâmica reprodutiva do camarão branco litopenaeus schmitti (Burkenroad, 1936) (Decapoda: Penaeidae) no litoral sul de Pernambuco. (Dissertação de mestrado. Universidade Federal Rural de Pernambuco)

Castro et al. (2020) Plano de Manejo da Área de Proteção ambiental do Delta do Parnaíba. ICMBIO 10-18

Carvalho, C. D. (2013). Crescimento e mortalidade do camarão branco Litopenaeus schmitti (Burkenroad, 1936) (Crustacea: Decapoda: Penaeidae) em ambiente natural e em confinamento. (Dissertação de mestrado. Universidade Federal Rural do Rio de Janeiro)

Cordeiro, C. A. M., Gomes, W. C. A., Silva, A. V. da, Dias, J. A. R., Paixão, P. E. G., Abe, H. A., Sousa, N. da C., Sampaio, D. de S., Silva, E. M. da, Fujimoto, R. Y., \& Barros, F. A. L. (2020). Effect of different smoking processes on the sensory and chemical attributes of two shrimps native to Brazil. Research, Society and Development, 9(11), e85591110460. https://doi.org/10.33448/rsd-v9i11.10460

Craveiro, Cecilia et al.(2019) Reproductive dynamics of the white shrimp Litopenaeus schmitti (Burkenroad 1936) in a beach seine fishery in northeastern Brazil. InvertebrateReproduction \& Development, 63, 111-121,

Correia Filho, F. L (2011). Projeto Cadastro de Fontes de Abastecimento por Água Subterrânea, estado do Maranhão: relatório diagnóstico do município de Tutóia / Francisco Lages Correia Filho, Érico Rodrigues Gomes, Ossian Otávio Nunes, José Barbosa Lopes Filho. - Teresina: CPRM - Serviço Geológico do Brasil, 
Research, Society and Development, v. 10, n. 10, e120101018554, 2021

(CC BY 4.0) | ISSN 2525-3409 | DOI: http://dx.doi.org/10.33448/rsd-v10i10.18554

Dos Santos, J. L. (2007). Pesca e Estrutura Populacional do camarãobranco Litopenaeus schmitti (Burkenroad, 1936) na região marinha e estuarina da baixada santista. São Paulo, Brasil.

Dos Santos, J. L., Severino-Rodrigues, E., \& André, M. (2018). Estrutura populacional do camarão-branco litopenaeus schmitti nas regiões estuarina e marinha da Baixada Santista, Sao Paulo, Brasil. Boletim do Instituto de Pesca, 34(3), 375-389.

Da Silva, P. S. M. (2018). Dinâmica Costeira do Litoral De Tutóia (MA), a partir da Análise Multitemporal de Imagens de Satélite e do Uso de Geoprocessamento (Doctoral dissertation, PUC-Rio).

Freire.C.D.S. et al. Estrutura populacional e morfometria do camarão branco Litopenaeus schimitti (Burkenroad,1936) capturado pela pesca artesanal em Sergipe, Brasil. Tropical Oceanography 47, n.1-16. 2019.

IBGE. 2010, < www.ibge.gov.br/cidadesat/topwindow.htm?1 >

Jornal Do Tempo. 2011. <http://jornaldotempo.uol.com.br/climatologia.html/Anajatuba-MA >

Lopes, M. (2012). Distribuição e dinâmica populacional dos camarões-rosa, Farfantepenaeus brasiliensis (Latreille, 1817) e F. paulensis (Pérez-Farfante, 1967) e do camarão-branco Litopenaeus schmitti (Burkenroad, 1936) (Decapoda: Dendrobranchiata: Penaeidae) no complexo baía-estuário de Santos-São Vicente, São Paulo, Brasil: subsídios científicos para a averiguação do período ideal de defeso. (Trse de Doutorado. Universidadae Estadual Paulista)

Machado, I. F, LFC Dumont \& F. D'Incao (2009).. Estagios de desenvolvimento gonadal e comprimento medio de primeira maturacao de femeas silvestres de camarao branco (Litopenaeus schmitti - Decapoda, Penaeidae) no Sul do Brasil. Atlantica, 31: 169-175.

Mendes Filho, O. da R., Figueiredo, E. S. A., Silva, K. C. de A., \& Cintra, I. H. A. (2020). Characterization of fishermen that integrate the fishing agreement in the middle river Araguaia region, Tocantins, Brazil. Research, Society and Development, 9(7), e529974516. https://doi.org/10.33448/rsd-v9i7.4516

Mossolin, E. C., \& Bueno, S. L. (2002). Reproductive biology of Macrobrachium olfersi (Decapoda, Palaemonidae) in São Sebastião, Brazil. Journal of Crustacean Biology, 22(2), 367-376.

Peixoto, S., Calazans, N., Silva, E. F., Nole, L., Soares, R., \& Frédou, F. L. (2018). Reproductive cycle and size at first sexual maturity of the white shrimp Penaeus schmitti (Burkenroad, 1936) in northeastern Brazil. Latin american journal of aquatic research, 46(1), 1-9.

Santos, M. D. C. F., Pereira, J. A., \& Ivo, C. T. C. (2006). A pesca do camarão branco Litopenaeus schmitti (Burkenroad, 1936) (Crustacea, Decapoda, Penaeidae) no nordeste do Brasil. Boletim Técnico Científico do CEPENE, 14(1), 33-58.

Santos, M.C.F. and Freitas, A.E.T.S. (2004), Estrutura populacional e pesca do camarão branco, Penaeus schmitti (Burkenroad, 1936) (Crustacea, Decapoda, Penaeidae) na lagoa Papari, município de Nísia Floresta (Rio Grande do Norte - Brasil), Boletim Técnico-Científico do CEPENE, 12(1), 23-42.

Santos, M. D. C. F., \& Menegon, M. (2010). Biologia e pesca do camarão Xiphopenaeus kroyeri (Heller, 1862) (Decapoda, penaeidae) e sua fauna acompanhante em São João da Barra, Rio de Janeiro, Brasil. Boletim Técnico Científico do CEPENE, 18(1), 31-48 